\title{
Cosmological quintessence accretion onto primordial black holes : conditions for their growth to the supermassive scale
}

\author{
P. S. Custódio and J.E.Horvath, \\ Instituto de Astronomia, Geofísica e Ciências Atmosféricas \\ IAG/USP, Rua do Matão, 1226, 05508-900 São Paulo SP, Brazil \\ Email: foton@astro.iag.usp.br
}

November 15, 2018

\begin{abstract}
In this work we revisit the growth of small primordial black holes (PBHs) immersed in a quintessential field and/or radiation to the supermassive black hole (SMBHs) scale. We show the difficulties of scenarios in which such huge growth is possible. For that purpose we evaluated analytical solutions of the differential equations (describing mass evolution) and point out the strong fine tuning for that conclusions. The timescale for growth in a model with a constant quintessence flux is calculated and we show that it is much bigger than the Hubble time. The fractional gain of the mass is further evaluated in other forms, including quintessence and/or radiation. We calculate the cosmological density $\Omega$ due to quintessence necessary to grow $\mathrm{BHs}$ to the supermassive range and show it to be much bigger than one. We also describe the set of complete equations analyzing the evolution of the $\mathrm{BH}+$ quintessence universe, showing some interesting effects such the quenching of the $\mathrm{BH}$ mass growth due to the evolution of the background energy. Additional constraints obtained by using the Holographic Bound are also described. The general equilibrium conditions for evaporating/accreting black holes evolving in a quintessence/radiation universe are discussed in the Appendix.
\end{abstract}




\section{Introduction}

Primordial black holes may be important in a number of situations. If $\mathrm{PBHs}$ formed in the early universe from primordial perturbations or some other mechanism, it is still not excluded that a population may survive. Some of them may even become the supermassive black holes that energize active galactic nuclei driving the powerful source of quasars, and may be a powerful sources of gravitational waves as well (see the review of M.Rees [1]).

The origin of supermassive black holes (SMBHs) remains a mystery, and since there are robust observational evidence for their existence with huge masses $\left(10^{6} M_{\odot}-10^{8} M_{\odot}\right)$, a big problem arises. How these objects can be formed in the universe? Were their initial masses very large from the beginning? There are several proposals for understanding these questions. One can suppose that the big black holes lurked in the AGNs can be formed from a primordial end point of the first generation of stars. A galactic nucleus could be populated by several massive bodies, and these objects fused into a only and single massive collapsed body, forming a central black hole. These models are discussed, for example, in Refs.[2] and references therein.

A second class of models (studied here) assume a huge growth of $\mathrm{PBHs}$ as a working hypothesis. Quite independently of the formation scenarios of small black hole seeds, the important question is whether they can grow to the supermassive range by means of accretion.

The analysis of the Hawking evaporation [3] of PBHs has leaded to conclude that PBHs initially larger than $M_{\text {haw }} \sim 10^{15} \mathrm{~g}$ could have chance to survive and contribute to a small fraction of the dark matter. A subset of PBHs may have grown to the supermassive range residing within the observed AGNs. In Ref. [4] we evaluated in detail the evolution and survival of PBHs taking into account evaporation and absorption from a radiation-dominated environment. We proved that all those $\mathrm{PBHs}$ within in the radiation-dominated era remained colder than the environment, and had a very small mass growth.

The simplest possibility for reaching the supermassive range is that $\mathrm{PBHs}$ formed in the early universe accreted mass from an energetic radiation environment (the Hawking evaporation was important only when their proper temperature became higher than the background temperature). If $\mathrm{PBHs}$ were massive enough, they could be colder than the environment, and accretion will drive their evolution. The accretion term may be constructed by multiplying the cross section for classical absorption of relativistic particles by the flux coming through the horizon. Taking into account the gravitational focusing of the incident radiation, this quantity is of order 


$$
\left(\frac{d M}{d t}\right)_{a b s}=\frac{27 \pi}{4} R_{g}{ }^{2} c \varrho_{r a d}(T) ;
$$

where the factor $27 \pi / 4$ describes the relativistic beaming of particles, see Zel'dovich and Novikov for details [5]. Since the gravitational radius is $R_{g}=2 G M / c^{2}$, this accretion is proportional to the second power of the black hole mass $M$. The formula above assumes that (in a first approximation) classical accretion does not affect the metric that describes the black hole itself. The issue is whether one can construct quintessence models which not only give the correct dynamics for the expansion, but also make PBHs grow to the supermassive range. This possibility is the subject of the following Sections.

\section{Quintessence models and Supermassive Black Hole growth}

There is now very good evidence that a dark energy is present in our universe and represents $\sim 0.7$ of the critical density. Today, the big challenge is understand the physical nature of this contribution. A crucial ingredient is that, contrary to the dark matter, dark energy must possess a negative pressure as required for an accelerating universe. Then, $\varrho_{\text {eff }}=(\varrho+3 P)<0$, implies $w<-1 / 3$, with $w=P / \varrho$ the velocity of sound.

At first sight, the most natural candidate is the cosmological constant. The cosmological constant can be viewed as a fluid exactly possessing $w=-1$. Since $\varrho+3 H(1+w) \varrho=0$, implies that $\varrho=$ constant. However, as it is well-known, explaining the dark energy with a cosmological constant runs into problems. The first, is that we need to generate a tiny ("unnatural") value at present epoch, to explain the magnitude of the acceleration of the universe. The second problem is the so-called "coincidence" problem. At high redshifts, say just after inflation $\left(z \sim 10^{28}\right)$, the radiation density was $\varrho_{\text {rad }} \sim 10^{61} \mathrm{GeV}^{4}$, while $\varrho_{\Lambda} \sim 10^{-47} \mathrm{GeV}^{4}$. This means that in order to have the correct amount of dark energy today, we need to fine-tune the initial conditions such that the ratio above was $10^{110}$ with a very high precision. This also seems very unnatural. These considerations leaded to consider that the dark energy is time-dependent. As is known from inflation models, a simple way of obtaining these features is to consider a minimally coupled scalar field $\varphi$ (termed quintessence). The latter is not necessarily the inflaton field, because we are at a much smaller energy scale, although the dynamical equations can be similar. Since the scalar field 
evolves with time, the equation of state itself varies with redshift, and it constitutes a very important observational challenge to measure this dependence [6].

It is beyond the scope of our work to analyze the whole features of the quintessential models, but instead we elaborate on the suggestion of Bean and Magueijo [7], who suggested an important growth of seed PBHs when a quintessence scalar field $\varphi$ is absorbed, dominating the accretion. The key new ingredient is the role played by the kinetic term $\left(\frac{\dot{\varphi}^{2}}{2}\right)$ in the flux onto the PBHs, which is absent in the case of accretion of pure radiation. They further showed that the flux at the horizon is given by $F_{\varphi}(t)=\frac{\dot{\varphi}^{2}}{2}(t)$ (in natural units), and the potential term $V(\varphi(t))$ drives the behavior of the kinetic term through the dynamical equations describing conservation and the cosmic expansion.

Type Ia supernovae data suggests that at $z<1$ the dark energy is dominant [8]. It is sure that for $z \gg 1$, the dark energy was not dominant, because eventually matter becomes important at these redshifts and earlier, for $z \sim 10^{4}$, radiation becomes more important. Then, we can conclude that, although we can not yet measure the dark energy contribution at $z \geq 1$, that contribution is subdominant but may be not negligible for the black hole growth problem from epochs earlier than present. We can view this in other form. The complete dynamics of quintessence plus radiation (and some black holes mixed as a secondary dark contribution) shows attractor solutions. The simplest models with quintessence show that it is very hard to have at, say, $z \sim 10$ and $\Omega_{D E}<<1$, while today $\Omega_{D E}(z \sim 1) \sim 0.7$. There are not "natural" solutions containing these features.

We shall describe a system considering that the energy and pressure of the quintessential field dominate the black hole contribution, and one simplified model arises from there.

A general model containing black holes + quintessence of the type studied here is described by

$$
S=\int d^{4} x \sqrt{-g}\left(\kappa R+L_{\varphi}+L_{\text {matter }}\right)
$$

where the quintessence scalar field has pressure and energy-density given by $P(t)=\frac{\dot{\varphi}^{2}}{2}-V(\varphi)$ and $\varrho(t)=\frac{\dot{\varphi}^{2}}{2}+V(\varphi)$ respectively.

We assume that our spacetime is asymptotically flat, consisting of this quintessential field and black holes (without charge or angular momentum) absorbing the energy of this field and neglect all other contributions, here $\kappa=1 / 16 \pi G$ is the gravitational constant. The total $T_{\mu \nu}$ does not need to be diagonal, even if the metric is diagonal in the beginning. The non-diagonal terms describe energy-flux at black hole horizons. 
The dynamics of the system is given by

$$
\begin{gathered}
\left(\frac{d M}{d t}\right)_{\text {growth }}=\frac{C M^{2}}{2} \dot{\varphi}^{2} ; \\
\ddot{\varphi}+3 H \dot{\varphi}+V^{\prime}=0 ; \\
H^{2}=\left(\frac{\dot{a}}{a}\right)^{2}=\frac{8 \pi}{3 M_{p l}^{2}}\left(V+\varrho_{p b h}\right)-\frac{K}{a^{2}} ; \\
\left(\frac{\ddot{a}}{a}\right)=-\frac{4 \pi}{3 M_{p l}^{2}}(\varrho+3 P) ;
\end{gathered}
$$

where $a(t)$ denotes the scale factor of expansion/contraction of the universe and $K=-1,0,+1$ describes the cosmic curvature. We shall evaluate physical effects in the flat model $K=0$ for simplicity, and assume that all the black holes started with the same mass, i.e. a single-mass scale initial function. We use natural units in which $C=27 \pi / M_{p l}{ }^{4}$. Note that eq.(4) is the conservation law for the quintessential field in the form $\dot{\varrho}+3 H(\varrho+P)=0$.

Since we have assumed from the scratch that PBHs do not dominate the dynamics of the universe $\varrho_{p b h}<<V(\varphi)$, analytical solutions for $\varphi(t)$ and its derivatives can be obtained without additional complications. It is obvious that, when this requirement breaks down, $\varphi(t)$ becomes a very complex function of $t$ and $M(t)$ and a numerical analysis is necessary.

Bean and Magueijo [7] have recently analyzed only one particular model of quintessence in which $V(\varphi)=\lambda \exp [\lambda \varphi]$, implying $\dot{\varphi}^{2} \propto t^{-2}$ within the slow-roll approximation. Within this model, in which the quintessence flux around the SMBH decreases with time, they claim that the black hole mass grows with growing time. However, the decreasing quintessence flux around these objects $\left(\propto t^{-2}\right)$ implies that the mass gain term decreases accordingly $\left(\dot{M} \propto M^{2} t^{-2}\right)$. In fact, we have proved that in the radiation-dominated era the growth of black holes is quenched when the background flux decreases with time as $t^{-2}$ or faster (this argument is generalized in the present work for quintessence accretion, see also Ref.[9]). It is important to gain some feeling about the behavior of simple solutions first, and we start with one of the simplest possibilities, namely that the flux of quintessence onto the black holes remains constant. 


\subsection{Constant quintessence-flux through the hori- zon event}

Since we are interested in evaluating the effect of the quintessential field on the black hole growth, we may start solving the particular case in which the kinetic term (and hence the flux) $F(t) \propto \dot{\varphi}^{2}$ is constant. If we set $\dot{\varphi}=$ constant $\equiv B$, eq.(4) yields

$$
\frac{\sqrt{4} 8 \pi \alpha(B)}{M_{p l}}=-\frac{d V}{V^{1 / 2}}
$$

with $\alpha(B)=\frac{\sqrt{48 \pi B}}{M_{p l}}$ and a slow-roll dynamics of the quintessence field holds. Solving for $V$ yields

$$
V(\varphi)=V_{0} \sqrt{1+\alpha(B)\left(\varphi-\varphi_{0}\right)} ;
$$

with $\alpha(B)=-\left(\frac{12 \pi B}{M_{p l}^{2}}\right)^{1 / 2}$.

The scalar field itself evolves as $\varphi(t)=\varphi_{0}+\sqrt{2 B}\left(t-t_{0}\right)$. Thus, the explicit solution for the evolution of the mass of the black hole is

$$
M(t)=\frac{M_{i}}{\left[1-\frac{54 \pi M_{i} B}{M_{p l}^{4}}\left(t-t_{i}\right)\right]} .
$$

For $t \gg t_{i}$, eq.(9) becomes

$$
M(t)=M_{i} \times\left[1-2.8 \times 10^{-22} h_{0}^{-1}\left(\frac{B}{\varrho_{c}}\right) \mu \tau\right]^{-1} ;
$$

where $\mu=\left(M_{i} / M_{\odot}\right), \tau=\left(t / t_{0}\right)$ and $\varrho_{c}\left(t_{0}\right) \sim 10^{-29} \mathrm{gcm}^{-3}$.

It is clear that the asymptotic value of the mass is much larger than the initial mass $M(t) \gg M_{i}$ if the denominator above satisfies

$$
0<\left[1-1-2.8 \times 10^{-22} h_{0}^{-1}\left(\frac{B}{\varrho_{c}}\right) \mu \tau\right]<\delta .
$$

with $\delta \ll 1$. The PBH mass actually diverges at $t_{a}\left(M_{i}\right)=\frac{M_{p l}^{4}}{54 \pi B M_{i}}$, numerically given by

$$
t_{a}\left(M_{i}, B\right) \sim 3.4 \times 10^{21}\left[\frac{\varrho_{c}\left(t_{0}\right)}{B}\right] \times\left[\frac{M_{\odot}}{M}\right] t_{0} ;
$$

with $t_{0} \sim 10^{17} h_{0}{ }^{-1} s$ and $\varrho_{c}\left(t_{0}\right) \sim 10^{-29} \mathrm{gcm}^{-3}$. This of course does not mean that a black hole will achieve an arbitrary large mass, but rather that before this happens, our approximations will break down and the condition $\dot{\varphi}=$ constant will not be satisfied. An analogous 
exercise in which the quintessence flux decreases as $F(t) \propto t^{-1}$ still produces a (slow but reasonable) growth over the cosmic time. However, for $F(t) \propto t^{-n}$, with $n>1$ the growth is quenched because the environment does not provide enough fuel as time goes by (see Section $3)$.

The next natural question is: how do the $\mathrm{PBHs}$ avoid the divergence of their masses in a "natural" way? For a generic quintessential field, but keeping the general slow-roll approximation for the dynamics of $\varphi$, we use the eqs.(3), (4) and (5) to obtain the mass of the black hole as a solution of a more complicated equation

$$
\left(\frac{d M}{d t}\right)=\frac{C M_{p l}^{2}}{48 \pi} \frac{M^{2}\left(V^{\prime}(\varphi(t))+\ddot{\varphi}\right)^{2}}{V(\varphi(t))+\varrho_{p b h}(t)} .
$$

The non-trivial effects of the presence of other black holes are now explicitly seen. In the limit $\varrho_{p b h}>V(\varphi)$ it is expected that the flux at the horizon decreases and the relation $F(t) \propto \dot{\varphi}^{2}$ ceases to be valid. On the other hand, the limit $V(\varphi) \gg \varrho_{p b h}$ leads to very simple solutions since this equation is separable (note that $\varrho_{p b h}(t)=M n_{p b h}$ ) and consists of growing solutions, holding for a finite time while $V(\varphi)>\varrho_{p b h}$.

\subsection{Evaluating the fractional $\mathrm{BH}$-mass variation for quintessence flux.}

Let us evaluate the fractional change in the black hole mass (born with an initial mass $M_{i}$ ) when the quintessence field alone fuels the growth.

Writing $\Omega_{\varphi}=\frac{\varrho_{\varphi}}{\varrho_{c}}$ and noting that the contribution of the kinetic term is $\frac{\dot{\varphi}^{2}}{2}$, then quite generally we have $\Omega_{\varphi}=\frac{\frac{1}{2} \dot{\varphi}^{2}+V(\varphi)}{\varrho_{c}}$.

Therefore, $\varrho_{c} \Omega_{\varphi} \sim \frac{\dot{\varphi}^{2}}{2}$, whenever $\frac{1}{2} \dot{\varphi}^{2}>V(\varphi)$. When the kinetic term dominates, we have $\frac{d M}{d t}=\frac{27 \pi}{M_{p l}^{4}} M^{2} \varrho_{c}(t) \Omega_{\varphi}(t)$, where $t=t(z)$. This equation is easy to solve. Integrating it from $t\left(z_{i}\right)$ until $t(z=0)$, and from $M_{i}$ until $M(t)$ yields

$$
\left(\frac{\Delta M}{M}\right) \sim \frac{27 \pi M_{i}}{M_{p l}^{4}} \int_{t\left(z_{i}\right)}^{t(0)} d t^{\prime} \varrho_{c}\left(t^{\prime}\right) \Omega_{\varphi}\left(t^{\prime}\right) .
$$

Since the variation of the $\Omega_{\varphi}$ is expected to be relatively small at low redshifts, and the quintessence term is known to amount $\sim 0.7$ today, we can always estimate the kinetic contribution of the quintessence as a function of the matter and radiation contributions. Neglecting as before the curvature term we may rewrite the eq.(14) above as 


$$
\left(\frac{\Delta M}{M}\right) \sim \frac{2.7 \pi M_{i}}{M_{p l}^{4}} \int_{z_{i}}^{0} d z^{\prime} \varrho_{c}\left(z^{\prime}\right) \frac{d t^{\prime}}{d z^{\prime}} ;
$$

which, after substituting the relations between $z$ and $t$, in terms of $H_{0}$, becomes

$$
\left(\frac{\Delta M}{M}\right)\left(z_{i}\right) \sim M_{i} H_{0} z_{i}
$$

which is linear in $z_{i}$, as expected. The formula above is a lower bound estimate, because we know that $\Omega_{\varphi}$ actually grows in that period. Then, it constitutes a minimal bound, when that variation of the latter is small.

If we further parametrize the flux as $F_{\varphi}=\varphi_{0}\left(t / t_{0}\right)^{n}$ for a generic index $n$, we can obtain from the condition $\Omega_{0}<1$

$$
\varphi_{0}<\sqrt{\varrho_{c}\left(t_{0}\right)} \sim 10^{-23} \mathrm{GeV}^{2} .
$$

Supposing that the kinetic term dominates over the potential one, and inserting this upper bound back into eq.(16) we obtain

$$
\left(\frac{d \mu}{d \tau}\right) \leq 10^{-22} \mu^{2} \tau^{n}
$$

where $\mu=\frac{M}{M_{\odot}}$ and $\tau=t / t_{0}$.

In these particular parametrized power-law models, $n$ can be positive, negative or null, and the results will differ for each case. Consider $n>0$ first. We can rise the following question: when will the net mass variation be relevant compared to the initial mass? Let us suppose that we want to obtain a fractional growth of $\sim 10^{8}$. Inverting the above relation, the cosmological time when this huge final mass will be achieved is

$$
t\left(\mu_{i}\right) \sim 10^{30 /(n+1)} \mu_{i}^{-1 /(n+1)} t_{0} ;
$$

in other words, if the conditions $\Omega_{\varphi}<1$ hold for future times and $n$ is constant and positive, only in the very remote future the scalar field accretion will be able to accrete $10^{8}$ times its initial mass. This future epoch of the universe will be coasting (provided $\Omega_{\text {total }}=1$ ), and dominated by big black holes that feed from the quintessential energy in a very slow way. For $n<0$, these conditions become much more severe, and we do not get any substantial growth unless very strong fine tuning had happened in the remote past. These conclusions are not likely to change for realistic (or more complete models) if we find the general background flux solutions given by the Friedmanns equations, since the fractional mass gain is 


$$
\left(\frac{\Delta \mu}{\mu}\right) \sim 10^{-22} \mu_{i} \int_{\tau_{i}}^{\tau} d \tau[f(\tau)] ;
$$

and $f(\tau)$ is a consistent solution of the complete set of equations. This is because it is very difficult to cancel the tiny coefficient $10^{-22}$, which arises quite generally in the consideration of the cosmological accretion. That is why we state that extreme $\mathrm{BH}$ growth can arise only in very peculiar situations, and why we believe that this is very difficult to achieve.

One can also invert the reasoning above and evaluate the density of radiation/quintessence in remote past which would have produced $\left(\frac{\Delta M}{M}\right) \gg 1$ and arrive to similar conclusions, namely that even given the most favorable conditions the quintessence would not be able to cause a huge growth of PBHs.

\section{Extreme fine-tuning for the growth of black holes immersed in a quintessence back- ground flux}

Using the critical mass (see [9] for discussion and references) as a tool let us show now that any flux of the form $F_{\varphi} \propto t^{-n}$, with $n>1$ does not lead to a substantial growth for any black hole, unless we require a strong fine-tuning between the formation-time and the initial mass of the black hole (with all other parameters kept fixed). This functional parametrization is specially suitable for most applications and permits to make a quick contact with standard cosmological evolutions.

The dynamical equation in the accretion regime is

$$
\left(\frac{d M}{d t}\right)=C M^{2} M_{p l}^{4}\left(t / t_{*}\right)^{-n} ;
$$

where $C$, and $t_{*}$ are constants. Plain integration yields

$$
\int_{M_{i}}^{M(\eta)} \frac{d M}{M^{2}}=\frac{C M_{p l}^{4}}{E_{*}} \int_{\eta_{i}}^{\eta} d \eta \eta^{-n} ;
$$

with $\eta=\left(t / t_{*}\right)$ and $E_{*}=t_{*}{ }^{-1}$.

The l.h.s. is just

$$
\frac{1}{M_{i}}-\frac{1}{M(\eta)}
$$

and the r.h.s. is 


$$
\frac{C M_{p l}{ }^{4}}{(n-1) E_{*}}\left[\frac{1}{\eta_{i}^{n-1}}-\frac{1}{\eta^{n-1}}\right] .
$$

Then, the solution $M(\eta)$ is

$$
\frac{1}{M(\eta)}=\frac{1}{M_{i}}\left[1-\frac{C M_{i} M_{p l}^{4}}{(n-1) E_{*}}\left(\frac{1}{\eta_{i}^{n-1}}-\frac{1}{\eta^{n-1}}\right)\right] .
$$

Considering $n>1$ we obtain for asymptotic times, i.e $\eta \gg \eta_{i}$

$$
M(\eta)=\frac{M_{i}}{\left[1-\frac{C M_{p l}{ }^{4}}{(n-1) E_{*}} \frac{M_{i}}{\eta_{i}^{n-1}}\right]} .
$$

It is clear that the denominator is much smaller than 1 (and positive) at the expense of a very strong fine tuning between $M_{i}$ and $t_{i}$. Let us evaluate a definite numerical example. Suppose that the growth factor must be of order $10^{8}$ as before, i.e the denominator

$$
1-\frac{C M_{p l}{ }^{4}}{(n-1) E_{*}} \frac{M_{i}}{\eta_{i}^{n-1}}=10^{-8} .
$$

Then, the formation time $t_{i}$ must have been very fine-tuned into the function above (in one part by several millions) in order to get a huge growth factor. If this did not happen then either the growth factor falls off quickly to 1 or the solution is unphysical. Note that this strong conclusion is very general, and it is independent of the origin of the flux through the black hole horizon, i.e. the result is valid for the radiation-dominated era, in which the flux was $c \varrho_{\text {rad }}(t) \propto t^{-2}$ or a quintessence-dominated era when $\dot{\varphi}^{2} \propto t^{-2}$ (see Ref.[9]).

\section{Further constraints on $\mathrm{PBH}$ growth from the Generalized Second Law and the Holo- graphic Bound}

The irreversible process of quintessence disappearance into a black hole is just a particular example of the Generalized Second Law of thermodynamics (hereafter GSL) formulated in the '70s by Bekenstein [10] and other following works [11, 12].

The GSL is the statement that the total entropy (black holes plus any other fields) never decreases with time

$$
\Delta S_{\text {total }}=\Delta S_{b h}+\Delta S_{\text {matter }}+\Delta S_{\varphi}+\Delta S_{\text {radiation }}>0 ;
$$


where

$$
S_{b h}(N, M) \sim 10^{77} N\left(M / M_{\odot}\right)^{2} ;
$$

counts for the entropy of $N$-black holes, $S_{\text {matter }}$ and $S_{\text {radiation }}$ is the normal matter and radiation entropies, and $S_{\varphi}$ is the entropy of the scalar field. A step forward towards the use of entropy as a tool in cosmology is the so-called Holographic Principle (HP). The Holographic Principle $[11,12,13]$, is the statement that all entropy/information contained in the physical universe can be attributed to its boundary. In numerical terms, the upper bound to total entropy (known as the Holographic Bound) is

$$
S_{\max }(t)<\frac{A(t)}{4 L_{p l}^{2}}
$$

where $A(t)$ is the boundary area of the physical system at $t$. If we assume that the HP can be applied to the cosmological models, then it is natural to choose the cosmological particle horizon to bound the area and write the maximum entropy as

$$
S_{h p}(t) \sim 8 \times 10^{121}\left(t / t_{0}\right)^{2} .
$$

If the inequality $S_{b h}(N, M)<S_{h p}(t)$ has to be satisfied, the accretion of quintessence should adjust in such a way that a black hole with initial mass $M_{i}$ may grow to values $M_{f}>10^{6} M_{\odot}$, without violating the Holographic Bound. Therefore, an upper bound to the local flux due to this global requirement is obtained.

It is clear that this energy input strongly depends on the initial mass of the PBH $M_{i}$ and needs to be very large if the black hole was initially small. In addition, if the accretion is very high, the black hole that was initially below the Holographic Bound will blow that bound at some point. Then, to keep these black holes below the Holographic Bound at any time, the constraint

$$
\dot{S}_{s m b h}(t)<\dot{S}_{h p}(t) ;
$$

must be satisfied, together with $S_{s m b h}\left(t_{i}\right)<S_{h p}\left(t_{i}\right)$ at the formation time $t_{i}$. When both conditions are required to hold, an absolute upper bound to the flux onto the $N$ accreting black holes can be established. It comes out to be $F_{\varphi}<\frac{M_{\odot}^{2}}{t_{0}^{2}}\left[\frac{10^{44} t}{C N M^{3}(t)}\right]$, or numerically

$$
F_{\varphi}(t)<4.8 \times 10^{-5} \frac{(t / s) h_{0}{ }^{2}}{\left(N / 10^{11}\right)\left(M / M_{*}\right)^{3}} g c m^{-2} s^{-1} ;
$$

with $M_{*} \sim 10^{8} M_{\odot}$. 
If the total number $N$ of black holes increases, the flux $F_{\varphi}(t)$ would be correspondingly smaller, in order to keep all black holes growing at moderate rate and the total entropy smaller than $A_{h p} / 4$.

A general and rigorous proof that the Holographic Bound can be applied to the cosmological models is lacking now, but if we proceed with these ideas we can get some insight about the local and global properties of black holes in realistic models of the universe, although the full meaning of eq.(44) is obscure yet. Some problems of these methods in cosmology are discussed in Refs.[12] and [13]. Upper bounds to black hole abundances have been obtained using these ideas [14].

Quite regardless of the use of the Holographic Bound, we expect that when the mass-energy of black holes is comparable to term $V(\varphi)$ in the Friedmann equation, the system response would be to deplete the quintessence flux onto the black hole horizon by decreasing $\dot{\varphi}^{2}$. A complete study of these features will be published elsewhere [15].

\section{Conclusions}

We have shown in this work that, generally speaking, the quintessence flux $\dot{\varphi}^{2}$ must decrease slower than $t^{-2}$ for PBHs to grow at all, and to stay constant or increase in time for substantial accretion to occur. This may be relevant for some PBHs achieving the SMBH condition in a short time, but only at the expense of fine-tuning in the parameters. Several options for the quintessence potential $V(\varphi(t))$ have been proposed in the literature, some of which can lead to high flux to feed PBHs (i.e. leading to large positive $\dot{M}$ ) for long times. One analytical solution of this class leading to $\dot{\varphi}^{2}=$ constant has been worked out as an example.

If the flux onto the black holes is constant, all them initially above the critical mass will grow until the term $\varrho_{P B H}$ approaches the energydensity in the cosmic background. From this point, these black holes would not keep growing at high rates, and the more complicated calculations have not been performed for this regime.

In Section 3 we showed that if the flux behaves as $F(t) \propto t^{-2}$, we can prove that all solutions with asymptotic growth $\left(M(t) \gg M_{i}\right)$ depend of an initial fine-tuning between the initial masses $M_{i}$ and their formation at $t=t_{i}$. Other fluxes that decrease more slowly (or even increase) than $t^{-2}$ may lead to very big black holes but only at $t \gg t_{i}$, a condition that is not favorable for quintessence accretion which is unimportant at high redshifts and thus does not have enough time to happen. 
In Section 4 we have invoked Holographic Bound arguments to show that a bound on the quintessence flux can be obtained quite generally, although these arguments would only hold if a rigorous proof of the existence of a bound is obtained. In spite of the theoretical efforts, this has not been achieved and therefore the limits must be considered as speculative.

It should be kept in mind that models others than the one discussed here can be constructed to produce a population of SMBHs starting from seed PBHs. For example, accretion in a brane-world high-energy phase has been recently studied by Guedens, Clancy and Liddle [16] and Majumdar [17], which show that a substantial growth in which $\dot{M} \propto M / t$ is allowed. It may be possible to arrive to the end of the high-energy phase with very massive black holes, although the full consequences of this type of scenarios are yet to be explored.

\section{Acknowledgments}

The authors wish to thank the São Paulo State Agency FAPESP for financial support through grants and fellowships. J.E.H. has been partially supported by $\mathrm{CNPq}$ (Brazil). We also acknowledge scientific advise from Prof. Elcio Abdalla.

\section{References}

[1] M.Rees, in 300 years of gravitation, (Cambridge University Press, 1986).

[2] C. Tyler,B. Jamus and D. Santos-Noble, astro-ph/0309008 (2003) and references therein.

[3] S.W. Hawking, Comm.Math. Phys.43, 199 (1975).

[4] P.S. Custódio and J.E. Horvath,Phys.Rev.D58, 023504 (1998).

[5] I.D. Novikov and Ya. B. Zel'dovich, Relativistic Astrophysics, V.2, (U. Chicago Press, 1985).

[6] P. Brax and J. Martin, astro-ph/0210533 (2002).

[7] R. Bean and J. Magueijo, Phys.Rev.D66, 063505 (2002).

[8] S. Perlmutter et al., Nature391, 51 (1998); A. Reiss et al., Astron.J.116, 1009 (1998).

[9] P.S. Custódio and J.E. Horvath, Gen. Relat. Grav.34, 1895 (2002).

[10] J. Bekenstein, Phys.Rev.D9, 3292 (1974) ; ibidPhys.Rev.D23, 287 (1981). 
[11] D. Bigatti and L. Susskind, TASI lectures on the Holographic Principle, hep-th/0002044 (2000).

[12] N. Kaloper and A. Linde, Phys.Rev.D60, 103509 (1999).

[13] R. Bousso, Rev.Mod.Phys.74, 825(2002).

[14] P.S. Custódio and J.E. Horvath, Class. Quant. Gra v.20, 813 (2003).

[15] P.S. Custódio and J.E. Horvath, in preparation.

[16] R. Guedens, D. Clancy and A.R. Liddle, Phys.Rev.D66,083509 (2002) .

[17] A.S. Majumdar, Phys.Rev.Lett.90, 031303 (2003).

[18] A. G. Riess et al, astro-ph/0402512 (2004).

\section{Appendix: the critical mass in radia- tion+quintessence environments}

A black hole surrounded by radiation/quintessence would be either absorbing energy or evaporating, according to the sign of the difference $T_{p b h}-T_{\text {environment }}$, just like any other thermodynamical system. Consideration of the complete differential equation describing the black hole mass evolution

$$
\left(\frac{d M}{d t}\right)=-\frac{A}{M^{2}}+C M^{2} F_{\text {total }}(t) ;
$$

is needed (where $F_{\text {total }}(t)$ represents the total flux of energy through the black hole horizon (that is, the addition of fluxes for any form of energy that falls through the black hole) at cosmic time $t$ ). For a given $F_{\text {total }}$ the condition $\frac{d M}{d t}=0$ defines a parameter (the critical mass $M_{c}(t)$ ) separating the absorbing and evaporating regimes. The most interesting cases arise when the flux is dominated by radiation or quintessence. We shall discuss the cases separately to highlight some interesting differences.

\subsection{Radiation-dominated environment}

The case of pure radiation $F_{\text {total }} \equiv F_{\text {rad }}(t)=c \varrho_{\text {rad }}(t)$ has been analyzed in [4]. By solving the algebraic equation $\frac{d M}{d t}=0$, the critical mass is found to be

$$
M_{c}\left(T_{\text {rad }}\right)=\frac{\theta}{T_{\text {rad }}(t)} \sim \frac{10^{26} g}{\left(T_{\text {rad }} / T_{0}\right)} ;
$$


where $\theta=M_{p l}\left(A / 27 \pi a_{*}\right)^{1 / 4}, \varrho_{\text {rad }}(T)=a_{*} T^{4}$ and $T_{0} \sim 3 K$ is the present temperature of the CMBR.

Then, if we ignore other possible sources of energy-matter to fuel their growth, we conclude that any black hole with mass $>10^{26} \mathrm{~g}$ is today in the absorption regime. The critical mass has evolved with time, it was $M_{c} \sim 10^{16} \mathrm{~g}$ when the universe was $t \sim 1 \mathrm{~s}$ old. Therefore, any $\mathrm{PBH}$ with initial mass greater than the latter value formed within the radiation-dominated era, was initially above the equilibrium condition, and the Hawking evaporation was irrelevant for it. Apart from fine details of the absorption process, the cross section is expected to be valid quite generally (see Refs. [4] and [9] for a full discussion).

\subsection{Quintessence-dominated environment}

If we consider the quintessential field as the dominating fuel, the balance equation for the black hole mass $\frac{d M}{d t}=0$ becomes

$$
\left(\frac{d M}{d t}\right)_{\text {total }}=-\frac{A}{M^{2}}+\frac{C M^{2}}{2} \dot{\varphi}^{2}
$$

In order to simplify the expressions, let us define the dimensionless ratio $A=\sigma M_{p l}^{4} \sim 1.1 \times 10^{-3}$. Thus, the critical mass for this case is expressed as

$$
M_{c}(t)=\frac{0.06 M_{p l}}{\Xi(t)^{1 / 4}} \sim 5.6 \times 10^{24} g\left[\frac{\dot{\varphi}^{2}}{2 \varrho_{c}\left(t_{0}\right)}\right]^{-1 / 4} ;
$$

where we have defined the dimensionless function $\Xi(t)=\frac{\dot{\varphi}^{2}}{2 M_{p l}}$.

The expression shows that if the quintessential density is of order of the critical density today, the corresponding critical mass is about $10^{25} \mathrm{~g}$. This is not very different than the value corresponding to pure radiation dominance, a fact directly related to the assumption $\Omega_{\varphi} \sim$ $\Omega_{\text {crit }}$.

Let us show how the constant flux case described in Section 2 looks like in terms of the critical mass. The critical mass is a constant given by

$$
M_{c}(t) \sim 5.6 \times 10^{24} g\left[\frac{B}{\varrho_{c}\left(t_{0}\right)}\right]^{-1 / 4} .
$$

Therefore, only initial masses greater than this value can be described by growing models with constant quintessence through the horizon. Fig. 2 displays the behavior of black holes in this situation according to their initial mass, see caption for details. More general models can be studied. For instance, let us briefly discuss the case of 
a power-law flux $F(t)=M_{p l}^{4}\left(t / t_{*}\right)^{n}$ with $t_{*}$ a reference time. In the case $n<0$, we obtain for the critical mass

$$
M_{c}(t) \sim(\sigma / 27 \pi)^{1 / 4} M_{p l}\left(t / t_{*}\right)^{|n| / 4} ;
$$

and this critical mass increases with increasing time. Since the ratio of the absorption term to the evaporation term is just

$$
-\frac{\dot{M}_{a b s}}{\dot{M}_{\text {evap }}}=\left(M / M_{c}\right)^{4}
$$

(valid for any form of the accreting flux) then, if we follow the evolution of a PBH mass whose initial mass $M_{i}$ was slightly larger than $M_{c}\left(t=t_{i}\right)$, then we can ignore the Hawking term on its evolution until the PBH mass crosses the critical mass curve. The error introduced to obtain the crossing time $t_{c}$ is small. Thus we can describe the PBH mass evolution as

$$
\frac{1}{M_{i}}-\frac{1}{M_{c}\left(t_{c}\right)}=\frac{27 \pi t_{*}}{(n-1)}\left[\frac{1}{\eta_{i}^{n-1}}-\frac{1}{\eta_{c}^{n-1}}\right] ;
$$

where $\eta_{i}=\left(t_{i} / t_{*}\right)$ and $\eta_{c}=\left(t_{c} / t_{*}\right)$. Since $t_{c} \gg t_{i}$ in general, the second term is always negligible.

\section{EDITOR: PLEASE PUT FIG.1 HERE!!!!!!!}

Solving eq.(22)for the crossing time yields

$$
t_{c}\left(M_{i}, t_{i}\right) \sim t_{*}\left(\frac{M_{i}}{M_{p l}}\right)^{4 / n}\left(\frac{\sigma}{27 \pi}\right)^{-1 / n}\left[1-\frac{27 \pi M_{i} t_{*}}{n-1}\left(t_{i} / t_{*}\right)^{1-n}\right]^{-4 / n} .
$$

Then, in a finite time, this PBH will achieve an instantaneous equilibrium at the critical mass crossing, and immediately proceed into the evaporating region.

In the case $n>0$, the critical mass is

$$
M_{c}(t) \sim M_{p l}(\sigma / 27 \pi)^{1 / 4}\left(t / t_{*}\right)^{-|n| / 4} .
$$

And this function decreases with increasing time. Suppose that a $\mathrm{PBH}$ has been formed with initial mass smaller than the critical mass at $t_{i}$. Then, for a long time this PBH will loss energy until its complete evaporation (if we ignore other quantum corrections likely to arise near the Planck scale). However, if its mass reached the critical mass at value $M_{c}\left(t_{c}\right)>M_{p l}$, then the black hole achieved an instantaneous equilibrium at $t_{c}$ (see below) and it will proceed into the absorbing 
region, from which it will be growing increasingly faster (Fig. 3). We note that at $M=M_{c}$ we have $\ddot{M}\left(M=M_{c}, t_{c}\right) \propto M^{2}\left(t / t_{*}\right)^{n-1}>0$, and it will be increasing for $n>1$. Now, if a PBH had already formed below the critical mass at $t_{i}$, and is not much smaller than it we can solve the mass differential equation to find the crossing time

$$
t_{c}\left(M_{i}\right) \sim t_{*}\left(\frac{\sigma}{27 \pi}\right)^{1 / n}\left(\frac{M_{p l}}{M_{i}}\right)^{4 / n} .
$$

This solution is numerically valid only when $t_{c}\left(M_{i}\right) \ll t_{\text {evap }} \sim$ $t_{0}\left(M_{i} / M_{\text {haw }}\right)^{3}$.

From $t_{c}\left(M_{i}\right)$, this black hole will be accreting and its mass will be growing. These considerations illustrate the role of the critical mass for the evaluation of the fate of PBHs. Any statement about growth/evaporation is necessarily time-dependent and must be carefully done to preclude a misunderstanding of the physical situation.

\section{EDITOR: PLEASE PUT FIG.2 HERE!!!!!!!}

In a completely generic case $F_{\text {total }}=F(t)$ we obtain

$$
\ddot{M}(M, t)=\left[\frac{2 A}{M^{3}}+2 C B M\right] \dot{M}(M, t)+C M^{2}(t) \dot{F}(t) ;
$$

and the fate of these black holes will depend on the sign of $\dot{F}(t)$ as expected. In general, $\ddot{M}\left(M=M_{c}, t\right)$ is not zero, and a similar conclusion holds for higher derivatives as well. Therefore, we conclude that very special conditions have to be fulfilled for black holes to evolve in equilibrium with the environment, that is, without losing or gaining mass.

\subsection{Quintessence/radiation environment}

If both radiation and quintessence are considered, then the critical mass becomes

$$
M_{c}(t) \sim \frac{10^{26} g}{\left(T_{r a d} / T_{0}\right)}\left[1+\frac{\dot{\varphi}^{2}}{\varrho_{r a d}}\right]^{-1 / 4} ;
$$

to be solved together with eqs.(4-6). In general, the behavior of $\varrho_{\text {rad }}$ would not be $\propto t^{-2}$, which happens only when the radiation dominates all forms of energy. In this case, the first derivative of $\dot{M}(M, t)$ at $M=M_{c}$ is given by

$$
\ddot{M}\left(M=M_{c}, t\right)=C M^{2}\left(4 \dot{T} T^{3}+\dot{\varphi} \ddot{\varphi}\right) ;
$$


and this term is in general not zero unless $\dot{\varphi} \ddot{\varphi}=-4 \dot{T} T^{3}$. Since radiation and quintessence would have to conspire for that, equilibrium of PBHs and the environment would not be possible. In scenarios where $V(\varphi)>>\varrho_{\text {rad }}$ PBHs can stay in equilibrium, actually one of these is the constant flux model already discussed $\dot{\varphi}^{2}=2 B=$ cte.

Now, we can evaluate the redshift dependence in these terms. In any way, $\varrho_{r a d}(z) \propto(1+z)^{4}$, regardless of whether the quintessence dominates. We can cast the eq.(47) above as

$$
M_{c}(z) \sim \frac{10^{26} g}{\left[(1+z)^{4}+\frac{1}{\varrho_{\text {rad }}(0)}\left(\frac{d \varphi}{d z}\right)^{2} \dot{z}^{2}\right]^{1 / 4}} .
$$

It is easy to conclude that if $\frac{1}{(1+z)^{4}} \frac{1}{\varrho_{\text {rad }}(0)}\left(\frac{d \varphi}{d z}\right)^{2} \dot{z}^{2} \propto(1+z)^{m-4}$ the following behaviors are possible. If $m>4$ the quintessence will determine the critical mass even at high redshifts. For $m<4$, the opposite case happens, and there will be a redshift $z_{l}$ from which the radiation will determine the critical mass. It will be true at $\left(1+z_{l}\right) \sim$ $\left[\left(\frac{d \varphi}{d z}\right)^{2} \dot{z}^{2}\right]^{1 / 4}$, evaluated at $z_{l}$ also. It depends (as it should be) on the time derivative of the quintessential field, and the complete solution must be solved when we choose some form of $V(\varphi)$. It is important note that when the term $V(\varphi)$ is relevant, the accretion into black holes can be null in some cases even when $\Omega_{\varphi}$ is big. We can write $\Omega_{\varphi}=$ $\frac{\frac{1}{2} \dot{\varphi}^{2}+V(\varphi)}{\varrho_{c}}$. Then, when the first term is important, the quintessence dominates and the black hole accretion (from it) is important also, but the inverse is not true. We can choose other models with $V(\varphi)$ leading to $\frac{1}{2} \dot{\varphi}^{2}=0$, and the quintessence dominates without some secondary effect onto black holes with any mass. The third point now is. When the form of $V(\varphi)$ leads to a non-null kinetic term, the magnitude of this term depends also on the magnitude of $V(\varphi)$ (obtained by solving the dynamics) and the accretion onto black holes will depends on the parameters that enter into $V(\varphi)$ - definition. Finally, the fourth point is that the z-dependence in $\Omega_{\varphi}(z)=\frac{\frac{1}{2} \dot{\varphi}^{2}(z)+V(\varphi(z))}{\varrho_{c}(z)}$ reflects in each term. Today we know that $\Omega_{\varphi}(z \sim 0) \sim 0.7$, but the attractor features of the solutions indicate that when $z \geq 1$, the magnitude of the terms above were not so much below the unity also, although the exactly dependence and values are not known yet. In terms of time, from $z=10$ to $z=0$ there was enough time to accretion into black holes, if the others conditions were satisfied together also. Moreover, there are indications that the quintessence varies with $z$ in a very slow way if at all, see [18]. 


\subsection{The behavior of $\ddot{M}\left(M=M_{c}, t\right)$}

An interesting related question is whether PBHs formed with $M_{i}=M_{c}$ can stay in equilibrium without evaporating or gain. We give a brief assessment of the general features of the solutions $M(t)$. Given the general form of $\dot{M}(M, t)$ we may express $\ddot{M}\left(M_{c}, t\right)$ in the following way

$$
\ddot{M}\left(M_{c}, t\right)=C M^{2}(t) \dot{F}(t) ;
$$

in terms of the mass and the behavior of the flux at its horizon. Since $M=M_{c}$, then it will be simplified to $C M_{c}^{2}(t) \dot{F}(t)$ and it is enough know the evolution of the critical mass and flux. The true behavior of each black hole mass is very complicated even in the simply cases above, but through the critical mass and its derivatives, we may infer the black hole mass at asymptotic times. Then, the algebraic sign and derivatives of $\ddot{M}\left(M_{c}, t\right)$ will determine the behavior of the solutions.

Applying these definitions to the constant flux case we obtain $\dot{M}(M=$ $\left.M_{c}, t\right)=0$ on the critical mass. The first derivative of $\dot{M}(M, t)$ is just $\frac{d}{d t}\left(-\frac{A}{M^{2}}+C M^{2} B\right)$, rewritten as

$$
\ddot{M}(M, t)=\left[\frac{2 A}{M^{3}}+2 C B M\right] \dot{M}(M, t) .
$$

Since $\ddot{M}\left(M=M_{c}, t\right)=0$, equilibrium is possible on the critical mass and the black holes will retain their initial masses. This result is valid as long as we ignore the radiation compared to the quintessence field. Note that all the higher derivatives of $\dot{M}(M, t)$ are null at $M_{c}$ only if the flux through the horizon is constant.

For the example mentioned in Section 3 of a power-law flux $F(t)=$ $M_{p l}^{4}\left(t / t_{*}\right)^{n}$. The critical mass is $M_{c}(t)=\frac{\alpha}{F^{1 / 4}(t)}$ with $\alpha=(A / C)^{1 / 4}$ and the second derivative $\ddot{M}$ reads

$$
\ddot{M}\left(M_{c}, t\right)=\frac{n C \alpha^{2} M_{p l}^{2}}{t_{*}^{n}}\left(t / t_{*}\right)^{n / 2-1} .
$$

From eq.(31) above, it is clear that we have a branching of the behavior for the solutions. The first branch corresponds to $n>2$ and in the limit $t \rightarrow \infty$ we obtain $\ddot{M}\left(M_{c}, t \rightarrow \infty\right)=\infty$. On the other hand, $n<2$ yields $\ddot{M}\left(M_{c}, t \rightarrow \infty\right)=0$ These cases are physically very different, because the function $\dot{M}(M, t)$ is related to the energy that crosses the horizon event, and its derivative is sensitive to changes in the asymptotic limit. The cases $0<n<2$ correspond to solutions 
showing moderate growth and the case $n>2$ to very fast growth of the black holes. The cases $n<0$ are the ones for which the critical mass grows, the behavior of black holes in this latter case is determined by the initial condition $M_{i}$. 
Fig.1. The critical mass for a constant quintessence flux. The critical mass is represented by the horizontal line labelled in the graph. A black hole may either grow or evaporate according to the value of its initial mass $M_{i}$ above or below the critical mass respectively. Instantaneous equilibrium of a black hole not growing nor evaporating is possible if its mass is precisely the critical value, as discussed in the text. Note that growing is expected to saturate when the primordial black hole density approaches the quintessence field density.

Fig. 2. The same as in Fig. 1 for a power-law quintessence flux of the form $F(t)=M_{p l}^{4}\left(t / t_{*}\right)^{n}$, with $n>0$. The black holes suffer a delay of their evaporation and may start to grow if they manage to cross the critical mass curve which decreases with increasing time. This case illustrates how different acceptable models of quintessence have to be analyzed individually to address the fate of PBHs possibly formed at early times .

Figure captions. 


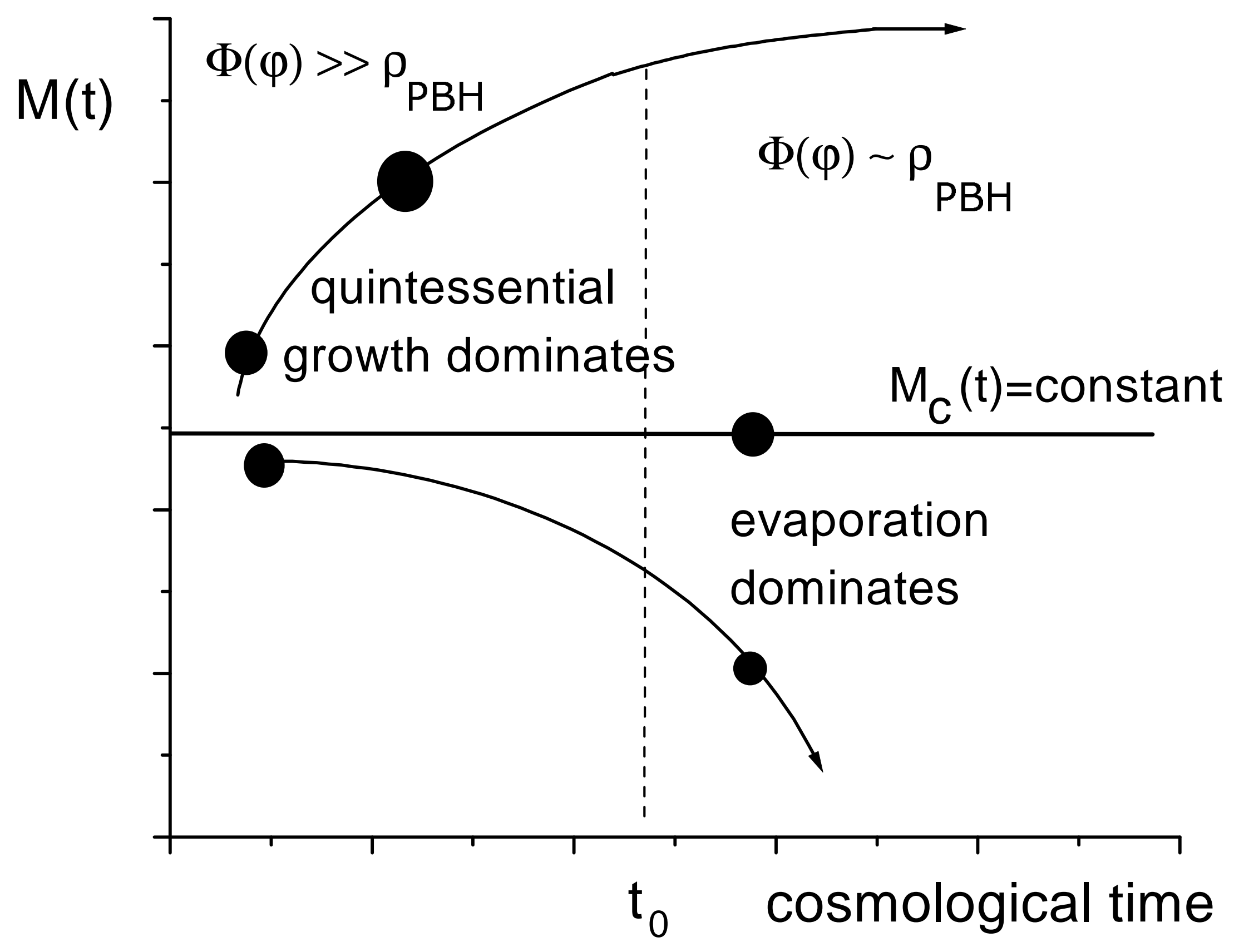

Fiaure 1 


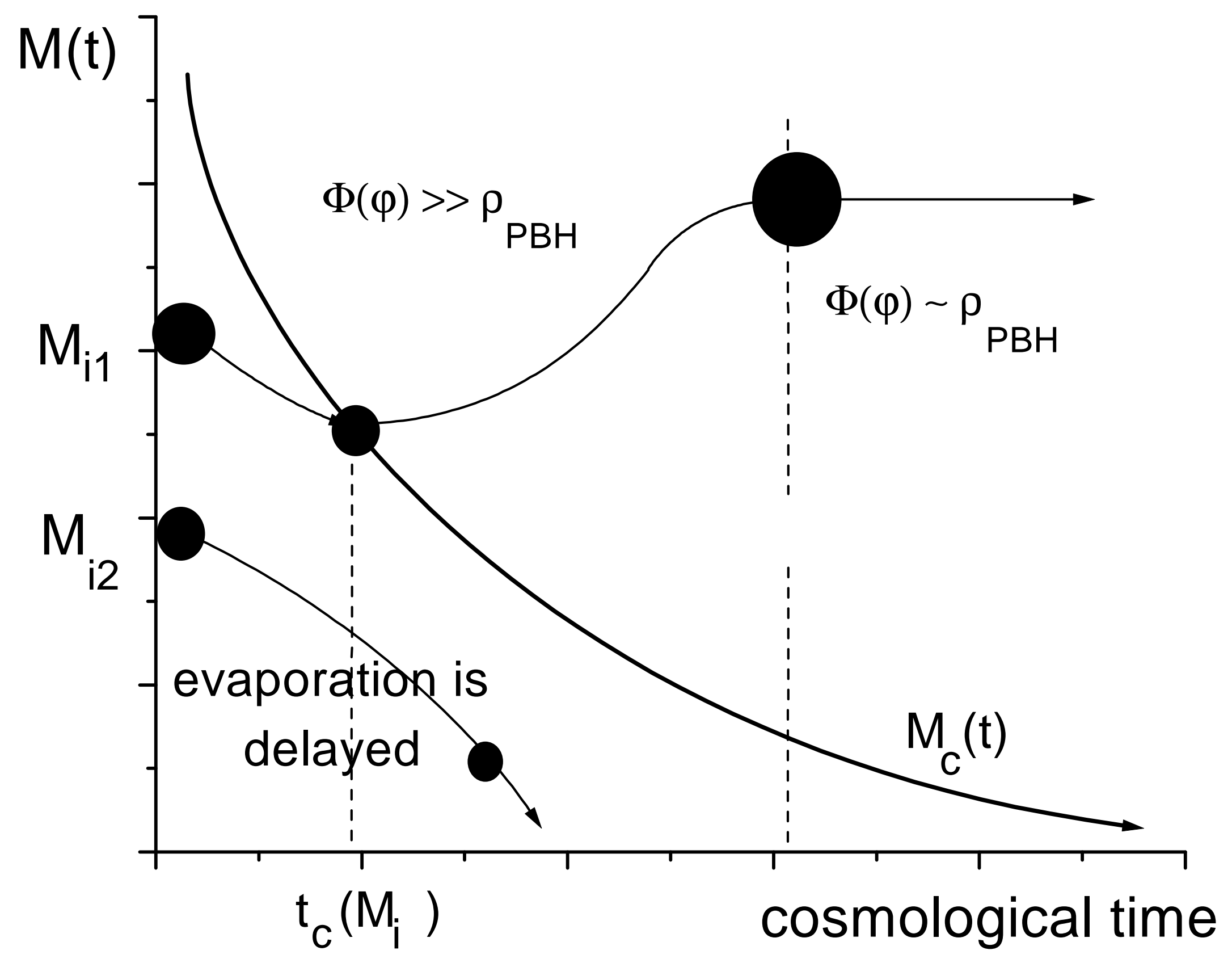

Figure 2 